Ischemic Attack (TIA). To improve the adherence of these patients, a complex individualised pharmacist intervention was designed and is being used in an ongoing study investigating the effect on medicines adherence and new stroke events. The present work is a subanalysis of this study.

Purpose To examine adherence-related issues in stroke/TIA patients identified by use of a complex pharmacist intervention including medicines review and motivational interviewing.

Materials and Methods The study is being performed at the Neurology Ward and the Emergency Ward, Odense University Hospital, where patients treated for TIA or acute ischemic stroke are randomised to a complex individualised pharmacist intervention or a control group. The pharmacist intervention consists of 3 components: 1) A medicines review focused on potential adherence-related problems followed by recommendations to the ward physicians 2) A motivational interviewing consultation where the content is based on issues raised by the patient 3) A follow-up telephone call one week after discharge with standardised adherence questions to uncover potential non-adherence.

Results Twenty-four patients received the pharmacist intervention. Among the topics covered, 7 potential adherence-related problems were identified. Four of the recommendations were accepted by the physicians, 2 were refused and there was no response to one. The issues most commonly addressed in the consultations were change of lifestyle (79\%), medicines management (67\%) and adverse reactions $(58 \%)$. Other issues included effectiveness of the medicines $(50 \%)$, adherence aids (42\%) and information about the disease (8\%). According to the standardised questions, one patient had adherence problems at the one-week follow-up phone call.

Conclusions A complex pharmacist intervention can be used to identify potential adherence-related problems in stroke patients.

No conflict of interest.

\section{CPC-008 ADHERENCE, TOLERABILITY AND QUALITY OF LIFE ASSESSMENT IN PATIENTS TREATED WITH TELAPREVIR}

doi:10.1136/ejhpharm-2013-000276.465

C Bustos Morell, JM Martínez-Sesmero, M García Palomo, N Labrador Andújar, FE Apolo Carvajal, AR Rubio Salvador, JJ Cía Lecumberri, P Moya Gómez. Hospital Virgen de la Salud, Pharmacy Department, Toledo, Spain

Background The addition of NS3/4 protease inhibitors to the standard of care treatment (SoCT) for genotype 1 hepatitis $\mathrm{C}$ (pegylated interferon and ribavirin) has increased the treatment response rate as well as the frequency and severity of adverse events (AEs). These may reduce the effectiveness or even cause the discontinuation of treatment.

Purpose To evaluate adherence, tolerability and quality of life $(\mathrm{QoL})$ in triple treatment patients (TT) (telaprevir + SoCT) in comparison with SoCT patients.

Materials and Methods Observational, prospective study performed in a 780-bed teaching hospital from February to September 2012. Prescription of TT was based on National Spanish Health System recommendations. A printed questionnaire was offered to patients (SoCT or TT) when they started on treatment and was given back three months later. The Questionnaire consisted of three parts: SMAQ (Simplified Medicines Adherence Questionnaire), Side Effects Profile Test (SEPT) (score from 1 to 5) and QoL Spanish version of the Chronic Liver Disease Questionnaire-Hepatitis C Virus (CLDQ-HCV) (score from 1 to 28). Statistical analyses were performed using SPSS 15.0 (non-parametric test).

Results A total of 53 hepatitis $C$ patients started drug treatment during the study (26 TT vs. 27 SoCT). We obtained 12 questionnaires on TT (46.1\% response rate, median age 52.4 years, $65.5 \%$ women) and 10 questionnaires of SoCT (37.0\% response rate, median age 49.3 years, $58.1 \%$ women). Only 2 TT (16.6\%) were non-adherent and 5 SoCT $(50.0 \%)(p=0.002)$. Data collected from SEPT showed a mean global score value of 2.2 in TT and 2.3 in SoCT $(p=0.356)$. The CLDQ-HCV mean global score was 15.9 in TT and 14.2 in SoCT ( $p=0.128)$.

Conclusions Better adherence in TT is probably due to patient expectations and highest motivation for the new drug. Perhaps, this also affects to similar groups rates in SEPT and CLDQ-HCV.

No conflict of interest.

\section{CPC-009 ADMINISTRATION OF DABIGATRAN REMOVED FROM THE CAPSULE}

doi:10.1136/ejhpharm-2013-000276.466

${ }^{1}$ A Kaneta, ${ }^{2} \mathrm{~T}$ Araki, ${ }^{1} \mathrm{M}$ Taira, ${ }^{3} \mathrm{~T}$ Aomori, ${ }^{1} \mathrm{D}$ Nagano, ${ }^{4} \mathrm{M}$ Arai, ${ }^{2} \mathrm{~T}$ Nakamura, ${ }^{4} \mathrm{M}$ Kurabayashi, ${ }^{2 K}$ Yamamoto. 'Gunma University Hospital, Pharmacy, Maebashi, Japan; 'Gunma University Graduate School of Medicine, Clinical Pharmacology, Maebashi, Japan; ${ }^{3} G$ unma University Graduate School of Medicine, Center for Medical Education, Maebashi, Japan; "Gunma University Graduate School of Medicine, Medicine and Biological Science, Maebashi, Japan

Background Dabigatran, an oral anticoagulant classified as a direct thrombin inhibitor, is used for the prevention of stroke and systemic embolism. However, it has limitations in its method of administration; dabigatran should not be removed from the capsule and administered through a tube because of its unstable bioavailability.

Purpose To report a case that required dabigatran to be administered through a tube after removal from the capsule.

Materials and Methods A 79-year-old Japanese male with normal hepatic and renal function was receiving warfarin for the prevention of systemic embolism due to atrial fibrillation. When he started S-1 treatment as an adjuvant treatment for gastric cancer, PT- and INR levels exceeded the scale. Because this elevation was thought to be due to the interaction between warfarin and S-1, warfarin was replaced with dabigatran. After switching anticoagulants, PT-INR and aPTT stabilised. Subsequently, however, the patient fell and experienced paralysis due to medullary damage. We tried to administer dabigatran through a tube after removal from the capsule while carefully monitoring the blood levels. Although the typical daily dose of dabigatran is $220 \mathrm{mg}$, the daily dose in the present case was set to $150 \mathrm{mg}$ in consideration of elevated blood concentration due to removal from the capsule. The dabigatran concentration $4 \mathrm{~h}$ after the first administration (peak) and before the second and third doses (trough) was measured by ultra-performance liquid chromatography/mass spectrometry.

Results The dabigatran concentration was 115.8, 62.45, and $80.05 \mathrm{ng} / \mathrm{mL} 4 \mathrm{~h}$ after the first administration and before the second and third doses, respectively, which is similar to data obtained in a clinical study using healthy Japanese volunteers. aPTT was 38-48 s. Conclusions We were able to administer dabigatran after removal from the capsule through a tube at two-thirds the regular dose and maintain a similar dabigatran blood concentration to that obtained in a clinical study through careful monitoring of dabigatran plasma levels.

No conflict of interest.

\section{CPC-010 ADVERSE EFFECTS AND EFFICACY OF ATROPINE $0.3 \%$ EYE DROPS IN PREMATURE INFANTS UNDERGOING SYSTEMATIC SCREENING FOR RETINOPATHY: AN OBSERVATIONAL STUDY}

doi:10.1136/ejhpharm-2013-000276.467

C Kowal, S Béchet, C Danan, L Caeymaex. Chi Creteil, 94000, Créteil, France 